\title{
Development of a broad-host synthetic biology toolbox for ralstonia eutropha and its application to engineering hydrocarbon biofuel production
}

\author{
Changhao Bi ${ }^{1,2}$, Peter Su ${ }^{1,3}$, Jana Müller ${ }^{1}$, Yi-Chun Yeh ${ }^{1,4}$, Swapnil R Chhabra ${ }^{1 *}$, Harry R Beller ${ }^{5}$, \\ Steven W Singer ${ }^{5}$ and Nathan J Hillson ${ }^{1^{*}}$
}

\begin{abstract}
Background: The chemoautotrophic bacterium Ralstonia eutropha can utilize $\mathrm{H}_{2} / \mathrm{CO}_{2}$ for growth under aerobic conditions. While this microbial host has great potential to be engineered to produce desired compounds (beyond polyhydroxybutyrate) directly from $\mathrm{CO}_{2}$, little work has been done to develop genetic part libraries to enable such endeavors.

Results: We report the development of a toolbox for the metabolic engineering of Ralstonia eutropha $\mathrm{H} 16$. We have constructed a set of broad-host-range plasmids bearing a variety of origins of replication, promoters, $5^{\prime}$ mRNA stem-loop structures, and ribosomal binding sites. Specifically, we analyzed the origins of replication pCM62 (IncP), pBBR1, pKT (IncQ), and their variants. We tested the promoters $\mathrm{P}_{\mathrm{BAD}}, \mathrm{T} 7, \mathrm{P}_{\mathrm{xyl} / \mathrm{PM}}, \mathrm{P}_{\text {lac }}$ vv5, and variants thereof for inducible expression. We also evaluated a T7 mRNA stem-loop structure sequence and compared a set of ribosomal binding site (RBS) sequences derived from Escherichia coli, $R$. eutropha, and a computational RBS design tool. Finally, we employed the toolbox to optimize hydrocarbon production in $R$. eutropha and demonstrated a 6-fold titer improvement using the appropriate combination of parts.
\end{abstract}

Conclusion: We constructed and evaluated a versatile synthetic biology toolbox for Ralstonia eutropha metabolic engineering that could apply to other microbial hosts as well.

Keywords: Broad-host, Synthetic biology, Ralstonia eutropha, Hydrocarbon, Chemolithoautotroph

\section{Background}

Chemoautotrophic "Knallgas" bacteria can utilize $\mathrm{H}_{2}$ / $\mathrm{CO}_{2}$ for growth under aerobic conditions, and have great potential to directly produce liquid fuels from $\mathrm{CO}_{2}$ and/or syngas [1,2]. Ralstonia eutropha ( $R$. eutro$p h a)$, the model bacterium of this class, can grow to very high cell densities $(>200 \mathrm{~g} / \mathrm{L})$ [3]. Under nutrient limitation, $R$. eutropha directs most of its carbon flux to the synthesis of polyhydroxybutyrate (PHB), a biopolymeric compound stored in granules. Under autotrophic growth conditions with $\mathrm{H}_{2} / \mathrm{CO}_{2}, R$. eutropha has been reported to synthesize $61 \mathrm{~g} / \mathrm{L}$ of PHB (representing 70\% of total cell weight) in $40 \mathrm{~h} \mathrm{[4]}$. With random

\footnotetext{
* Correspondence: swap.chhabra@gmail.com; njhillson@lbl.gov

'Physical Biosciences Division, Lawrence Berkeley National Laboratory, Berkeley, CA 94720, USA

Full list of author information is available at the end of the article
}

mutagenesis and relatively simple engineering, PHB and related polyhydroxyalkanoate polymers have been produced in R. eutropha on industrial scales [3].

While $R$. eutropha has great potential to be engineered to produce desired compounds (beyond PHB) directly from $\mathrm{CO}_{2}$, little work has been done to develop genetic part libraries to enable such endeavors. Although suicide vectors have been used to generate inframe deletions and point mutations in R. eutropha [5], and previously reported broad-host range expression systems [6] may be transferable to R. eutropha, to date, the only established inducible expression system for $R$. eutropha has been a pBBR1-derived vector with a $\mathrm{P}_{\mathrm{BAD}}$ promoter [7]. Here, we have initiated the development of a synthetic biology toolbox to enable complex metabolic engineering applications in $R$. eutropha H16. We evaluated a variety of vectors, promoters, 5 ' mRNA 
stem-loop sequences, and ribosomal binding sites (RBSs), and rationally mutated and engineered these genetic components to improve and diversify their function in R. eutropha. We then applied the resulting toolbox to engineer and optimize a hydrocarbon production pathway. Taken together, this work develops and demonstrates the engineering utility of a plasmid-based toolbox for R. eutropha.

\section{Results}

Broad-host vector evaluation and engineering

Three broad-host-range plasmid vectors were selected as starting points for the construction of new plasmid-based expression systems for $R$. eutropha: 1) pCM62, a lowcopy-number plasmid within the IncP incompatibility group [8]; 2) pBBR1MCS, a medium-copy-number plasmid [9]; and 3) pKT230, a high-copy-number plasmid

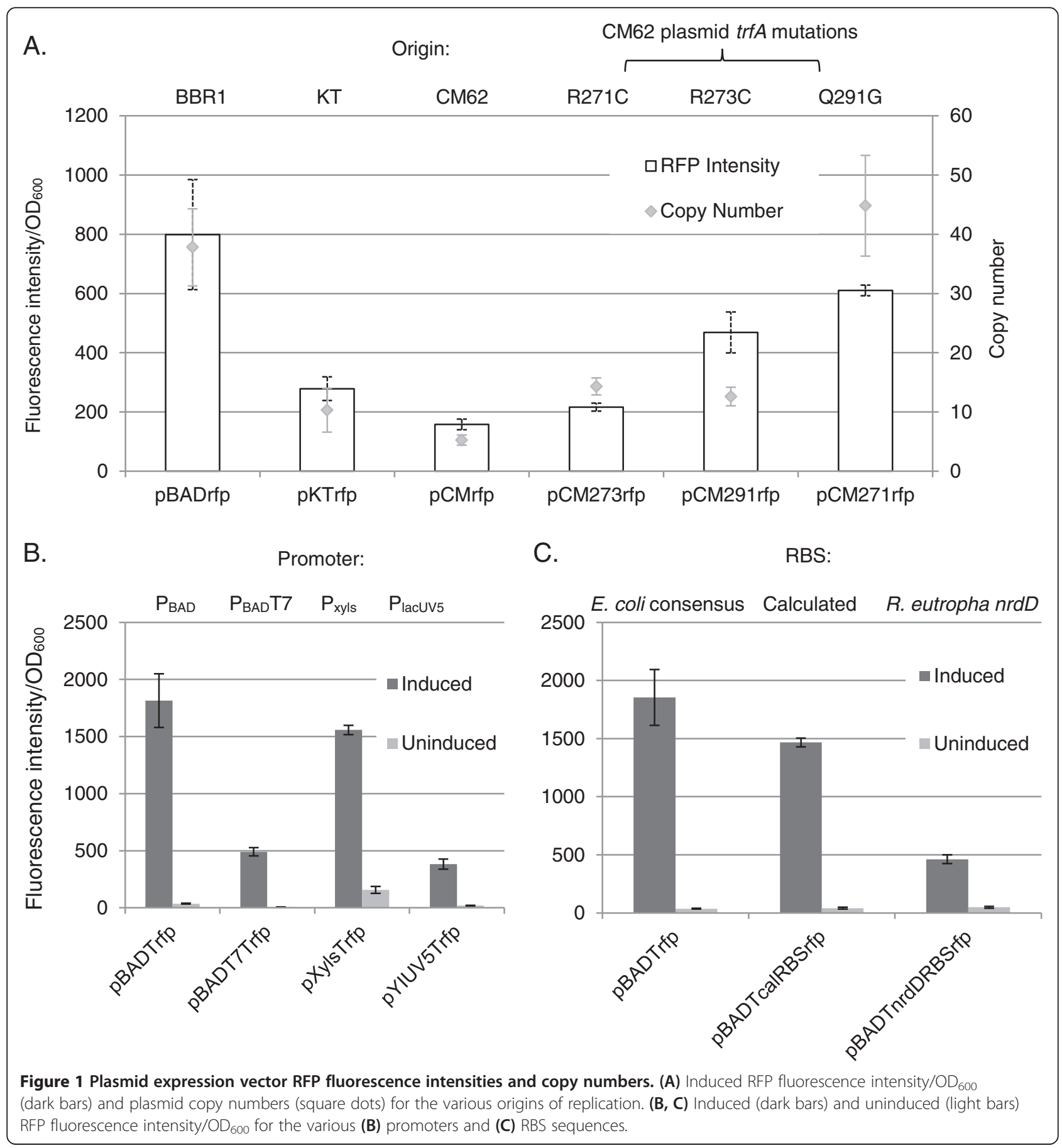


Table 1 Strains and plasmids used in this study

\begin{tabular}{|c|c|c|}
\hline$\underline{\text { Strain }}$ & Description & Reference \\
\hline R. eutropha H16 & R. eutropha wildtype strain currently classified as Cuparividus necator & ATCC 17669 \\
\hline R. eutropha $\mathrm{H} 16 \triangle 2303$ & $\mathrm{H} 16 \triangle\left(\mathrm{H} 16 \_\right.$A0459-0464, H16_A1526-1531); $\Delta$ beta ox; mutant is deficient in native-oxidation & [11] \\
\hline E. coli DH10B & $\mathrm{F}^{\prime}$ pro $A^{+} B^{+}$lac19 $\triangle$ lacZM15/ fhuA2 $\triangle\left(\right.$ lac-proAB) glnV galR (zgb-210::Tn10) Tet ${ }^{\mathrm{S}}$ endA1 thi-1 $\Delta$ (hsdS-mcrB)5 & NEB \\
\hline E. coli S17 & E. coli host strain for transconjugation & [12] \\
\hline Plasmid & Description & Reference \\
\hline$\overline{\mathrm{pCM}} 62$ & Broad host-range plasmid IncP group; $a m p^{R}$, tet ${ }^{R}$ & {$[8]$} \\
\hline PBBR1MCS & Broad host-range plasmid compatible with IncQ, IncP, IncW, and colE1; kan ${ }^{R}$ & [9] \\
\hline pKT230 & Broad host-range plasmid IncQ group; $k a n^{R}$ & [10] \\
\hline pBADrfp & pBBR1MCS derivative; $P_{B A D \_r f p}$ & [13] \\
\hline pBbE8c-RFP & colE1; PBAD_rfp; $\mathrm{cm}^{R}$ & {$[14]$} \\
\hline pBbA8a-RFP & 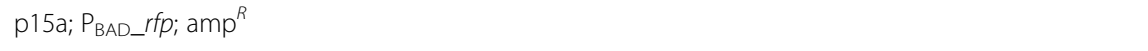 & [14] \\
\hline pKTrfp & 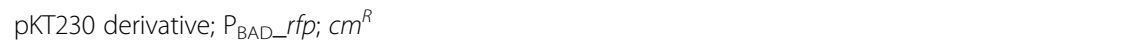 & This study \\
\hline pCMrfp & pCM62 derivative; $P_{\text {BAD_rfp; }} a m p^{R}$, tet $^{R}$ & This study \\
\hline pCM271rfp & pCMrfp with TrfA R271C mutation & This study \\
\hline pCM273rfp & pCMrfp with TrfA R273C mutation & This study \\
\hline pCM291rfp & pCMrfp with TrfA Q291G mutation & This study \\
\hline pBADTrfp & pBADrfp derivative; $\mathrm{P}_{\mathrm{BAD} \_} T 7$ stem-loop_rfp & This study \\
\hline pXylsTrfp & 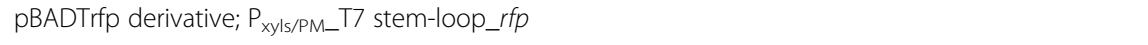 & This study \\
\hline pUV5Trfp & pBADTrfp derivative; $\mathrm{P}_{\text {lacUV5_T7 stem-loop_rfp }}$ & This study \\
\hline plUV5Trfp & pBADTrfp derivative; lacl ${ }^{q} ; P_{\text {lacuv5_T7 stem-loop_rfp }}$ & This study \\
\hline pTetTrfp & pBADTrfp derivative; $\mathrm{P}_{\text {tet_}} T 7$ stem-loop_rfp & This study \\
\hline pProErfp & pBADTrfp derivative; $P_{\text {proE_- }} r f p$ & This study \\
\hline pProSrfp & pBADTrfp derivative; $P_{\text {pros_ }} r f p$ & This study \\
\hline pBADT7Trfp & 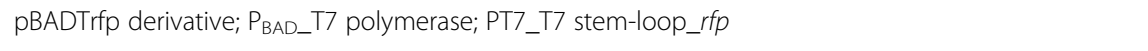 & This study \\
\hline pYIUV5Trfp & pBADTrfp derivative; lacY lacla; $P_{\text {lacuv5_T7 stem-loop_rfp }}$ & This study \\
\hline pBADTcalRBSrfp & pBADTrfp derivative; $\mathrm{P}_{\mathrm{BAD} \_} \mathrm{T7}$ stem-loop_calRBS $\mathrm{rp}$ _rfp & This study \\
\hline pBADTnrdDRBSrfp & pBADTrfp derivative; PBAD_T7 stem-loop_nrdDRBS_rfp & This study \\
\hline pKTTrfp & pKT230 derivative; $\mathrm{P}_{\mathrm{BAD} \_} \mathrm{T7}$ stem-loop_rfp; $\mathrm{cm}^{R}$ & This study \\
\hline pCMTrfp & pCM62 derivative; $\mathrm{P}_{\mathrm{BAD}} \mathrm{T}$ 7 stem-loop_rfp & This study \\
\hline pCM271Trfp & pCM62 derivative; pCMTrfp with TrfA R271C mutation & This study \\
\hline pCM271TcalRBSrfp & pCM271rfp derivative; $P_{B A D \_} T 7$ stem-loop_calRBS ${ }_{\text {rfp_rfp }}$ & This study \\
\hline pBADTHC & pBADrfpT derivative; PAD_T7 stem-loop_aar_adc & This study \\
\hline PKTTHC & pKTrfp derivative; PAD_T7 stem-loop_aar_adc $_{\text {BAD }}$ & This study \\
\hline PCMTHC & pCM62 derivative; $\mathrm{P}_{\mathrm{BAD}} \mathrm{T7}$ stem-loop_aar_adc & This study \\
\hline pCM271THC & pCM271rfp derivative; $P_{\text {BAD_T7 stem-loop_aar_adc }}$ & This study \\
\hline PBADHC & pBADrfp derivative; $P_{\text {BAD_aar_adc }}$ & This study \\
\hline pXylsTHC & pBADrfp derivative; $\mathrm{P}_{\mathrm{xyl} / \mathrm{s} / \mathrm{PM} \_T 7 \text { stem-loop_aar_adc }}$ & This study \\
\hline pYIUV5THC & pYIUV5Trfp derivative; Placuv5_T7 stem-loop_aar_adc & This study \\
\hline pBADTcalRBSHC & pBADTHC derivative; $P_{\text {BAD_T7 stem-loop_calRBS aar_aar_calRBS }}$ adc_adc & This study \\
\hline pBADTnrdDRBSHC & pBADTHC derivative; $P_{\text {BAD_T7 stem-loop_nrdDRBS_aar_nrdDRBS_adc }}$ & This study \\
\hline pCM271TcalRBSHC & pCM271rfp derivative; $P_{\text {BAD_T7 }}$ stem-loop_calRBS ${ }_{\text {aar_aar_calRBS }}$ adc_adc & This study \\
\hline
\end{tabular}

within the IncQ group containing the RSF1010 origin [10]. The kanamycin-resistance selection marker within pKT230 was replaced with a chloramphenicol-resistance marker to enable co-selection with pCM62 and pBBR1MCS-derivative plasmids. An inducible $r f p$ expression cassette containing a $\mathrm{P}_{\mathrm{BAD}}$ promoter [7], an E. coli 
consensus RBS, $r f p$, and a double terminator was incorporated into all three plasmid types. While none of the resulting plasmids were successfully electroporated into $R$. eutropha, they were all successfully transconjugated. As shown in Figure 1A, pBADrfp (the pBBR1MCSderivative, see Table 1) provided the highest induced RFP expression level, while pKTrfp and pCMrfp had lower expression levels. Plasmid pBADrfp (BBR1 origin, kanamycin resistance) co-propagated stably with pKTrfp (KT origin, chloramphenicol resistance) or pCMrfp (CM62 origin, tetracycline resistance) in $R$. eutropha (data not shown).

To increase the copy number of the pCMrfp plasmid, previously reported site-directed mutations were made to the $\operatorname{trf} A$ gene [15]. While putatively high-copynumber pCMrfp mutants (TrfA positions 251, 254 and 234) were not successfully transconjugated and established in $R$. eutropha, possibly because high-copynumber plasmids are not well tolerated in $R$. eutropha [16], medium-copy-number pCMrfp mutants (TrfA R271C, R273C and Q291G) were established. The mutant pCMrfp plasmids pCM271rfp, pCM273rfp, and pCM291rfp were measured to have higher RFP expression levels than pCMrfp (Figure 1A). To determine the absolute copy numbers of the pCMrfp plasmid variants, qPCR was performed using $R$. eutropha colonies as the source of the template (Figure 1A). pCM271rfp was determined to have the highest copy number $(44.8 \pm 8.5$ copies per cell) among the pCMrfp variants. pCM273rfp and pCM291rfp both had higher copy numbers than pCMrfp.

\section{T7 stem-loop structure evaluation}

A T7 stem-loop structure [17] was inserted upstream of the RBS of the $r f p$ gene on plasmid pBADrfp, yielding pBADTrfp. Introducing the T7 stem-loop structure into pBADTrfp increased RFP expression (1814 \pm 236 RFP intensity/OD, Figure 1B) by approximately 2-fold over pBADrfp levels (798 \pm 185 RFP intensity/OD) (Figure 1A).

\section{Inducible promoter system evaluation and engineering}

In addition to $\mathrm{P}_{\mathrm{BAD}}$, several other inducible promoter systems were evaluated in $R$. eutropha. Various repressor or activator genes along with their respective operators and promoters were inserted into pBADTrfp, replacing araC/ $\mathrm{P}_{\mathrm{BAD}}$. As shown in Figure $1 \mathrm{~B}$, the $\mathrm{P}_{\mathrm{BAD}}$ (pBADTrfp) and $\mathrm{P}_{\mathrm{xyls} / \mathrm{PM}}$ ( $\mathrm{pXylsTrfp)}$ promoter systems provided the highest RFP expression upon induction. This is the first demonstration that the $\mathrm{P}_{\mathrm{xyls} / \mathrm{PM}}$ promoter system is functional in $R$. eutropha. The T7 promoter controlled by $\mathrm{P}_{\mathrm{BAD}}$-induced T7 polymerase (pBADT7Trfp), although only providing modest RFP expression upon induction, had very little expression in the absence of induction. $\mathrm{P}_{\text {lacUv5 }}$ (pUV5Trfp and pIUV5Trfp), $P_{\text {tet }}$ (pTetTrfp), and $P_{\text {pro }}$ (pProErfp and pProSrfp) systems did not show inducible expression in $R$. eutropha (Additional file 1: Figure $\mathrm{S} 1$, and data not shown). The $\mathrm{P}_{\text {lac }} /$ lacI system has been reported previously not to be functional in $R$. eutropha [18]. Genomic sequence comparison between $R$. eutropha $\mathrm{H} 16$ and $E$. coli revealed that $R$. eutropha lacks the galactose permease gene lacY. This permease facilitates the transportation of lactose as well as the $\mathrm{P}_{\text {lac }}$ inducer IPTG into E. coli [19]. A lacY gene codon-optimized for $R$. eutropha expressed from a constitutive promoter was incorporated into pIUV5Trfp, yielding pYIUV5Trfp. As shown in Figure $1 \mathrm{~B}$ and Additional file 1: Figure S1, the incorporation of the lacY gene into pYIUV5Trfp enabled the IPTG-inducible expression of RFP from $\mathrm{P}_{\text {lacUV5, }}$, although the expression level is low compared to those of $\mathrm{P}_{\mathrm{BAD}}$ and $\mathrm{P}_{\mathrm{xyls} / \mathrm{PM} \text {. }}$

Cross-induction perturbation assays were performed to test if the chemical inducers L-arabinose $\left(\mathrm{P}_{\mathrm{BAD}}\right), m$ toluic acid $\left(\mathrm{P}_{\mathrm{xyls} / \mathrm{PM}}\right)$, and IPTG $\left(\mathrm{P}_{\text {lacUV5 }}\right)$ affect the performance of their non-cognate promoter systems (Table 2). For the most part, the three chemical inducers did not significantly perturb their non-cognate promoter systems. For example, the induction of Pxyls/PM by $1 \mathrm{mM} m$-toluic acid retained $95.4 \%$ and $98.0 \%$ of normal levels, respectively, when $1 \mathrm{mM}$ IPTG or $0.1 \%$ L-arabinose were added. An important exception is that $1 \mathrm{mM}$ $m$-toluic acid negatively impacted the induction of $\mathrm{P}_{\mathrm{BAD}}$ by $0.1 \%$ L-arabinose to about $60 \%$ of normal levels. However, when the $m$-toluic acid concentration was reduced from $1 \mathrm{mM}$ to $0.5 \mathrm{mM}$, the induction of $\mathrm{P}_{\mathrm{BAD}}$ by $0.1 \%$ L-arabinose remained at $91.7 \pm 1.6 \%$ of normal levels.

\section{Ribosomal binding site sequence evaluation}

Three RBS sequences were evaluated to compare their translation initiation efficiencies in R. eutropha: 1) an E. coli consensus RBS sequence (pBADTrfp), 2) a RBS calculator [20] designed RBS sequence (pBADTcalRBSrfp), and 3) the $R$. eutropha nrdD RBS sequence (pBADTnrdDRBSrfp). RBS calculator parameters were specified towards designing a strong RBS sequence for $R$. eutropha, with the setting at "max", provided pBADTrfp RBS region context. The $E$. coli consensus RBS provided the highest RFP expression levels (Figure $1 C$ ), while the computationally designed RBS

Table 2 Promoter cross-induction test

\begin{tabular}{cccc}
\hline Promoter & \multicolumn{3}{c}{ Non-cognate inducer added } \\
\cline { 2 - 4 } & L-arabinose & $\boldsymbol{m}$-toluic acid & IPTG \\
\hline PBAD & $(100 \%)^{\mathrm{a}}$ & $61.5 \pm 3.9 \%$ & $105.0 \pm 28.8 \%$ \\
Pxyls/PM & $98.0 \pm 2.7 \%$ & $(100 \%)^{\mathrm{a}}$ & $95.4 \pm 9 \%$ \\
PlacUV5 & $97.4 \pm 3.3 \%$ & $91.0 \pm 0.24 \%$ & $(100 \%)^{\mathrm{a}}$ \\
\hline
\end{tabular}

${ }^{a}$ Cognate inducer alone.

Observed florescence intensity relative to cognate inducer alone. 
provided medium to high RFP expression, and the native $R$. eutropha $n r d D$ RBS provided the lowest RFP expression levels.

\section{Applying the toolbox to hydrocarbon production optimization}

The synthetic biology toolbox was iteratively applied to optimize hydrocarbon production in $R$. eutropha. Genes encoding acyl-ACP reductase (aar) and aldehyde decarbonylase $(a d c)$ [21] were codon optimized for $R$. eutropha and synthesized (GenScript). These two synthesized genes were incorporated as an operon into the R. eutropha expression vectors developed above. The first set of constructed vectors (pBADTHC, pKTTHC, pCMTHC, and pCM271THC) was designed to determine the impact of plasmid origin of replication on hydrocarbon product titer (Figure 2A). Independent of the origin of replication, expressing the aar-adc hydrocarbon pathway in R. eutropha H16 resulted predominantly in the production of pentadecane (from palmitic acid) and heptadecene (likely from oleic acid [1]). The pBADTHC plasmid (pBBR1 origin) achieved the highest combined (pentadecane + heptadecene) titer, whereas the pCMTHC plasmid (pCM62 origin) produced the lowest. The pCM271THC plasmid (mutant pCM271 origin) was able to achieve a combined hydrocarbon titer comparable to that of PBADTHC, albeit with a more balanced pentadecane:heptadecene ratio. Removing the T7 stem-loop structure from pBADTHC, yielding plasmid pBADHC, did not significantly affect hydrocarbon titer, with the combined hydrocarbon for both reaching approximately $1000 \mu \mathrm{g} / \mathrm{L}$ (Additional file 1: Figure S2). The next set of constructed vectors (pXylsTHC and pYIUV5THC) was designed to determine the impact of the promoter on hydrocarbon product titer (Figure 2B). Of the three promoters tested, $\mathrm{P}_{\mathrm{BAD}}$ achieved the highest levels of hydrocarbon production, while $\mathrm{P}_{\mathrm{xyls} / \mathrm{PM}}$ and $\mathrm{P}_{\mathrm{la}}$ cUV5 only achieved low hydrocarbon titers (Figure 2B). The final set of constructed vectors (pBADTcalRBSHC and pBADTnrdDRBSHC) was designed to determine the impact of the RBS sequence on hydrocarbon product titer (Figure 2C). The E. coli consensus RBS sequence (pBADTHC) (tandem placement 5' of both aar and $a d c$ ) achieved the highest combined hydrocarbon titer, while the calculated (calRBS ${ }_{\text {aar_- }} a a r$ and calRBS ${ }_{\text {adc_- } a d c)}$ and the $R$. eutropha $n r d D$ (tandem placement 5' of both aar and $a d c$ ) RBSs produced about $70 \%$ and $30 \%$ as much, respectively. The calculated RBSs achieved the most balanced pentadecane:heptadecene ratio. Since changing the pBBR1 origin/E. coli consensus RBS sequence combination (pBADTHC) to either mutant pCM271 origin/E. coli consensus RBS sequence (pCM271THC) or pBBR1 origin/calculated RBS sequence (pBADTcalRBSHC) combinations did not dramatically reduce combined hydrocarbon titers, but produced a more

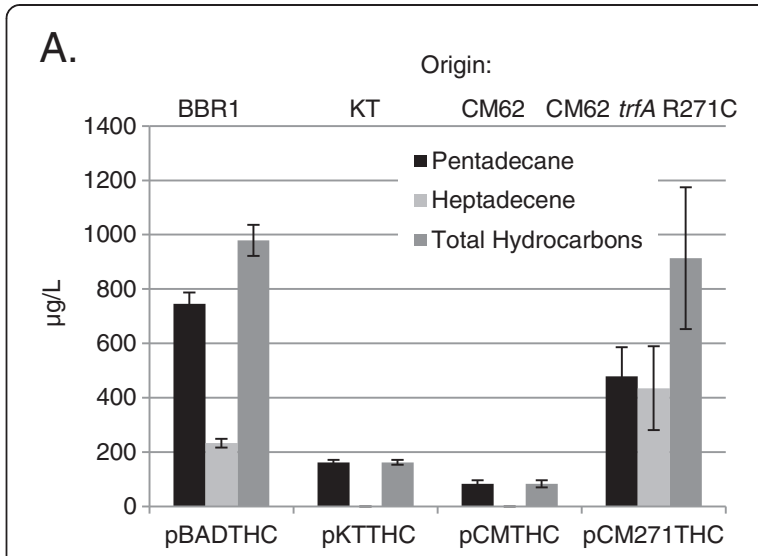

B.

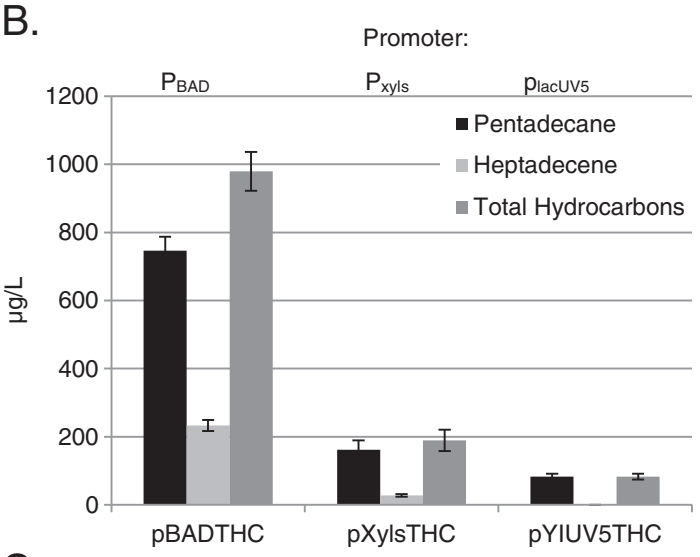

C.

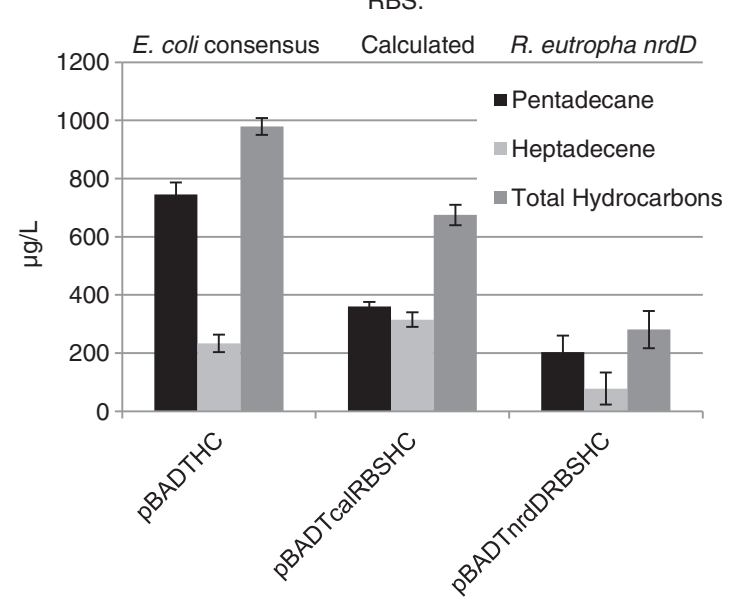

Figure 2 Hydrocarbon titers for the various $(A)$ origins of replication, (B) promoters, and (C) RBS sequences; (dark bars) pentadecane, (light bars) heptadecene, and (grey bars) combined.

balanced pentadecane:heptadecene ratio, we constructed plasmid pCM271TcalRBSHC to evaluate the hydrocarbon titer of the mutant pCM271 origin/calculated RBS sequence combination. Surprisingly, pCM271TcalRBSHC achieved a 6-fold improvement in combined hydrocarbon titer ( $6 \mathrm{mg} / \mathrm{L}$, Figure 3 ) relative to pBADTHC, 
the highest titer construct using previously established $R$. eutropha expression system components [7]. Furthermore, pCM271TcalRBSHC achieved a 100-fold improvement over the lowest production plasmids, pCMTHC and pYIUV5THC (Figure 4).

\section{Relationship between RFP expression level and hydrocarbon production titer}

To visually assess the relationship between toolbox component effects on RFP expression level and hydrocarbon production titer, the pBADT expression cassette (consisting of a pBBR1origin, PBAD promoter, T7 stemloop sequence, and E. coli consensus RBS) was used as a normalization point of reference. The reference plasmids pBADTrfp and pBADTHC were normalized to $100 \%$ relative RFP intensity and hydrocarbon production titer, respectively. The relative percentages for RFP intensity and hydrocarbon production titer for other expression cassette plasmid pairs are presented in Figure 4. Relative RFP fluorescence intensity appears to only slightly positively correlate linearly with relative hydrocarbon titer.

\section{Discussion}

Our reporter construct results revealed a dynamic range of $r f p$ expression levels for the genetic parts in the toolbox (Figure 1) and rapidly identified those parts that are nonfunctional in $R$. eutropha H16 $\Delta 2303$ (Additional file 1: Figure S1). We then investigated the impact of the toolbox on hydrocarbon production titer in $R$. eutropha, and compared the resulting titers with corresponding $r f p$ levels across the various expression configurations. In our system, RFP fluorescence intensity weakly correlated with hydrocarbon titer (Figure 4), suggesting that RFP fluorescence intensity is only a modest predictor of hydrocarbon titer, and should not generally be assumed to be a surrogate for pathway genes. This is perhaps not surprising,

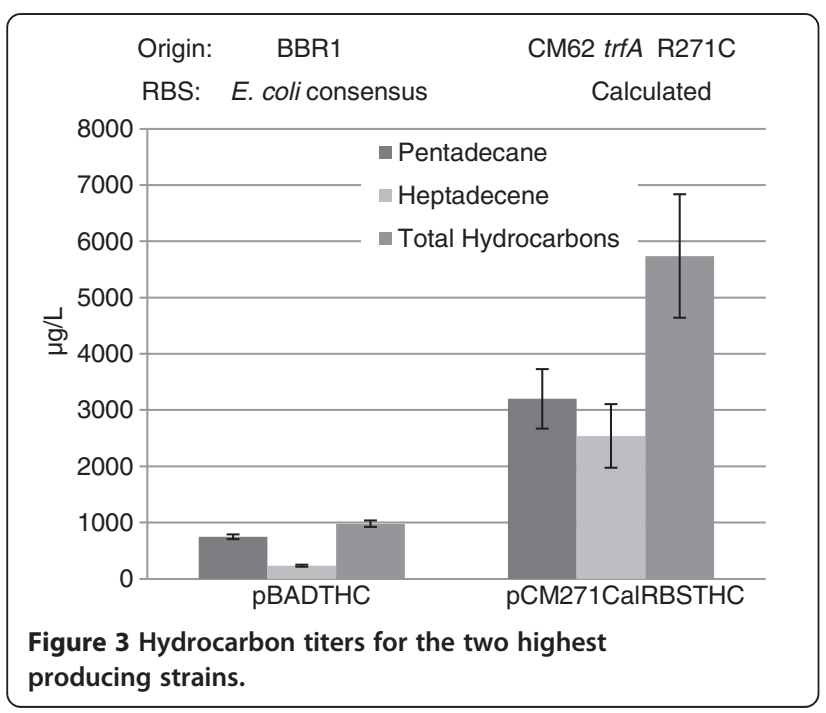

given that: 1) even RFP and GFP expression may only weakly correlate with each other over identical expression configurations [22], and thus RFP expression may not be a reliable reporter of hydrocarbon production pathway expression; and 2) product titer may not linearly or monotonically relate to pathway expression (i.e., more pathway expression does not necessarily translate to higher product titers [23]). While future work will be required to explain the mechanism behind the unexpected 6-fold improvement in combined hydrocarbon titer for pCM271TcalRBSHC over pBADTHC (Figures 3 and 4 ), we suspect that subtle differences in pathway expression may have serendipitously resulted in substantial titer increases. As such, what is more important than simply maximizing expression of pathway components is the capability to fine tune expression with sufficient granularity to resolve pathway bottlenecks and alleviate toxicity effects. The work reported here specifically contributes to this important capability.

We were surprised to observe that including the pCM271 vector and/or the calculated RBS parts in the hydrocarbon pathway expression construct resulted in balanced levels of pentadecane:heptadecane production in contrast with all other configurations for which the ratio was skewed predominantly to pentadecane (Figures 2 and 3). While the underlying mechanism for the relationship between pentadecane:heptadecane skew on expression configuration remains to be elucidated, it is interesting that using various components of the toolbox developed here affected not only overall product titers, but also product ratios.

Although we did not leverage this capability here, it is worth noting that since plasmid pBADrfp (BBR1 origin, kanamycin resistance) co-propagated stably with pKTrfp (KT origin, chloramphenicol resistance) or pCMrfp (CM62 origin, tetracycline resistance) in $R$. eutropha, it would be possible to engineer a multi-gene metabolic pathway across two plasmid vectors in the same cell (for example, see [24]). Furthermore, promoter cross-induction test results (Table 2) suggest that separate inducible promoters could be used to independently tune the expression of different portions of the pathway. These capabilities will play important future roles in engineering and optimizing more complex metabolic pathways in R. eutropha.

Metabolic engineering efforts often focus on a small set of microbial hosts, such as E. coli and Saccharomyces cerevisiae, simply because there are many established genetic and heterologous gene expression tools available for them. This select group of model microbes, however, may have limited utility for many industrial applications of interest. On the other hand, microbial hosts with metabolic capabilities and growth conditions well suited for specific industrial applications (like $R$. eutropha, which can function as a chemolithoautotroph), but with 


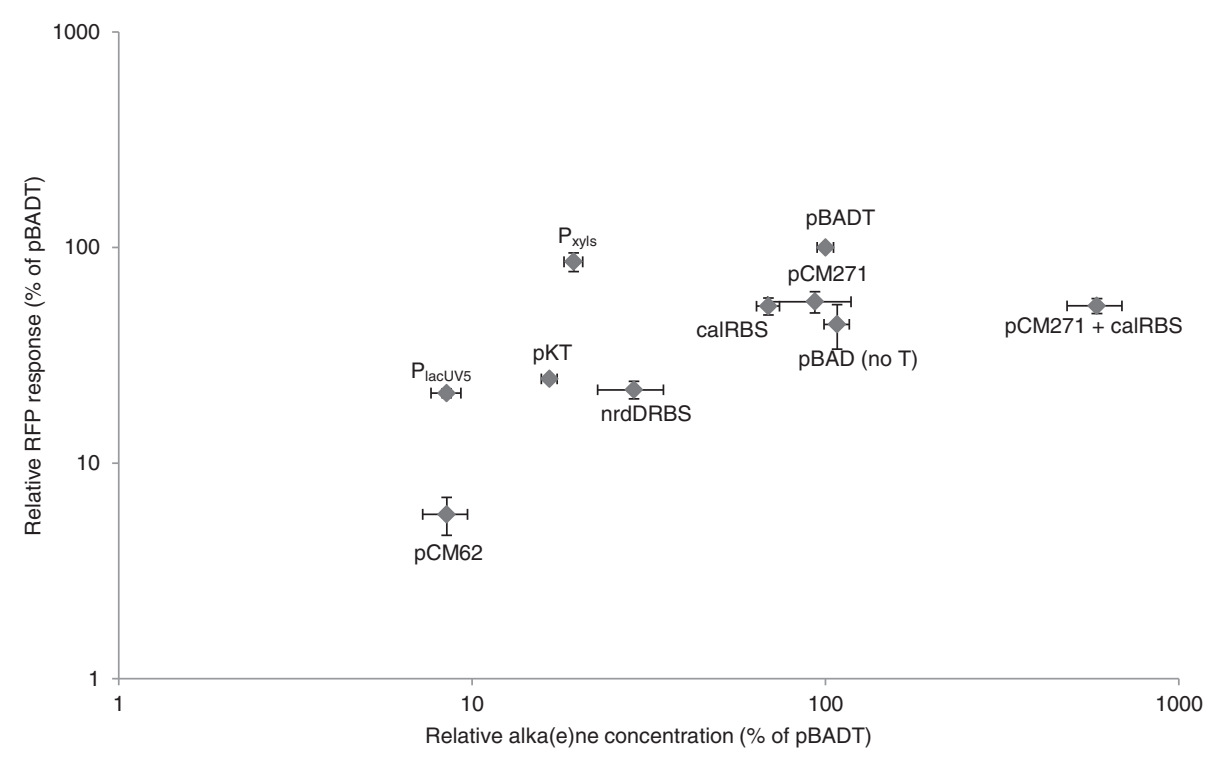

Figure 4 RFP expression levels and hydrocarbon production titers. Values normalized to reference plasmids pBADTrfp and pBADTHC. pCM62: pCMTrfp and pCMTHC. PlacUv5: pYIUV5Trfp and pYIUV5THC. pKT: pKTTrfp and pKTTHC. Pxyls: pXylsTrfp and pXylsTHC. nrdDRBS: pBADTnrdDRBSrfp and pBADTnrdDRBSHC. calRBS: pBADTcalRBSrfp and pBADTcalRBSHC. pCM271: pCM271Trfp and pCM271THC. pBADT: pBADTrfp and pBADTHC. pBAD (no T): pBADrfp and pBADHC. pCM271 + calRBS: pCM271TcalRBSrfp and pCM271TcalRBSHC.

limited genetic tools, are extremely challenging and time-consuming to metabolically engineer, and developing new genetic tools for specific microbes of interest can be entire research efforts in and of themselves [25]. Here, we have developed and deployed the toolbox for the metabolic engineering of $R$. eutropha. From the outset, we designed our efforts with broad-host range applicability in mind so that we could readily apply the same tools to other microbial hosts of interest. For example, the RSF1010 origin-derived pKT plasmids developed here are able to replicate in a wide range of Gram-negative bacteria (e.g., Enterococci) as well as the phylogenetically distant cyanobacteria, which are also important hosts of interest for biofuels production [10]. Plasmids within the IncP incompatibility group (including $\mathrm{pCM} 62$ ) and the $\mathrm{P}_{\mathrm{xyl} / \mathrm{PM}}$ promoter system have been demonstrated to function in Pseudomonas putida $[26,27]$. All vectors reported here contain mob genes to bolster efficacy across a wide range of bacteria. We envision that the broad-host range toolbox developed here will serve as a turnkey foundation for developing a robust set of metabolic engineering tools for other microbes of interest by simplifying and streamlining the process of screening for functional expression systems that operate within the microbe of interest. Building upon this vision, the toolbox could be exploited to screen metabolic pathway performance across multiple microbial hosts, through the direct transfer of constructs (e.g., pCM271TcalRBSHC) to microbes with overlapping functional expression systems. This approach, especially when coupled with no or low leakage inducible promoters (e.g., $\mathrm{P}_{\mathrm{BAD}} \mathrm{T7}$, Figure $1 \mathrm{~B}$ ), may be particularly effective for identifying microbial hosts that are tolerant to target or pathway intermediate compounds that are toxic to model microbes such as E. coli.

\section{Conclusions}

In this work, we have developed a toolbox for the metabolic engineering of $R$. eutropha H16, comprising six vectors spanning three compatibility groups, four promoter systems responding to three chemical inducers, a T7 5' mRNA stem-loop structure, and three RBSs. The major contribution of this work is that through increasing genetic regulatory part diversity, we have extended the dynamic range and tunable granularity of gene expression available for R. eutropha. We have demonstrated the value of the developed toolbox by increasing combined pentadecane and heptadecene hydrocarbon production titer 6-fold over that achievable with previously available gene expression tools and 100-fold over that achieved by our lowest producing engineered strains. Due to the broad-host range of the selected vectors and mobilized plasmid construction, this toolbox has a great potential to be applied to other microbial hosts for metabolic engineering purposes.

\section{Materials and methods}

\section{Bacterial cultivation}

$R$. eutropha H16 (ATCC 17669), R. eutropha H16 $\Delta 2303$ [11], E. coli DH10B (NEB) and S17 [12] were grown at $30^{\circ} \mathrm{C}$ in lysogeny broth (LB). Kanamycin 
(50 mg/L for E. coli; $200 \mathrm{mg} / \mathrm{L}$ for R. eutropha), ampicillin $(50 \mathrm{mg} / \mathrm{L})$, chloramphenicol $(30 \mathrm{mg} / \mathrm{L})$, tetracycline $(10 \mathrm{mg} / \mathrm{L})$ and/or gentamycin $(10 \mathrm{mg} / \mathrm{L})$ were added to the medium as appropriate.

\section{Plasmid construction}

With the exceptions of pBADTrfp, pBADTcalRBSrfp, pBADT7Trfp, pBADTnrdDRBSrfp, pXylsTrfp, pKTrfp, pCMrfp, pCM271rfp, pCM273rfp, and pCM291rfp (see Additional file 1), plasmids were assembled with the CPEC or Gibson methods [28,29], and corresponding DNA assembly protocols and DNA oligo primers were designed with j5 and DeviceEditor [30,31]. DNA templates were PCR-amplified with Phusion high-fidelity polymerase (Thermo Scientific). PCR products were gel purified before CPEC or Gibson assembly. The assembled plasmids were either transformed into E. coli DH10B, screened by colony PCR [32], sequence validated (Quintara Biosciences), and then transformed into $E$. coli S17 or directly transformed into R. eutropha H16 $\Delta 2303$, screened by colony PCR, and then sequence validated. Plasmids were then transconjugated from $E$. coli S17 into $R$. eutropha H16 $\Delta 2303$ as previously described [12].

\section{Strain and plasmid availability}

The strains and plasmids used in this study are listed in Table 1. All strains and plasmids developed here, along with their associated information (e.g., annotated GenBankformat sequence files, sequence validation trace files, DeviceEditor design files, and j5 design output files), have been deposited in the public instance of the JBEI Registry [33] (https://public-registry.jbei.org; entries JPUB_001171JPUB_001230) and are physically available from the authors and/or addgene (http://www.addgene.org) upon request.

\section{RFP fluorescence assay}

To measure the fluorescence intensity of RFP (monomeric mRFP1, maturation < 1 hour ) [34] expressed from each type of plasmid vector, single colonies were picked and inoculated into LB seed-culture tubes supplemented with kanamycin, chloramphenicol, gentamycin, or tetracycline, as appropriate. $100 \mu \mathrm{L}$ of each overnight seed culture was inoculated into a fresh $5 \mathrm{~mL} \mathrm{LB}$ culture tube supplemented with the appropriate antibiotic and inducer (IPTG, L-arabinose, $m$-toluic acid, or tetracycline) and grown at $30^{\circ} \mathrm{C}, 200 \mathrm{rpm}$ for 48 hours. $100 \mu \mathrm{L}$ of each cell culture tube was then added to a separate well in a 96-well clear-bottom plate (Corning: No. 3631) and RFP fluorescence was measured with a Safire (Tecan) microplate reader using an excitation wavelength of $585 \mathrm{~nm}$ and an emission wavelength of $620 \mathrm{~nm}$. OD 600 was also measured for each well immediately thereafter to calculate the RFP fluorescence intensity/OD 600 ratio reported for this assay.

\section{Plasmid copy number assay}

Single colonies (serving as templates) were picked, suspended in water, and then boiled for 10 minutes. Primers (pcmF and pcmR, phaZF and phaZR; Additional file 1) to amplify 400 to $500 \mathrm{bp}$ fragments of $r f p$ and phaZ respectively, were designed with Clone Manager 8.0. qPCR reactions were performed with a StepOnePlus Real-Time PCR System (Life Technologies) with Maxima SYBR Green/Fluorescein qPCR Master Mix (Thermo Scientific) as recommended by the manufacturers. Three biological replicates, with two technical replicates each, were performed for each plasmid type. Absolute plasmid copy numbers were determined using the CT difference between the plasmid-borne $r f p$ gene and the single copy chromosomal phaZ gene.

\section{Inducer dose response assay}

For each plasmid type, $10 \mu \mathrm{L}$ of overnight LB cell culture was inoculated into each of 4 separate 24-well clear-bottom plate wells containing $1 \mathrm{~mL}$ fresh LB supplemented with a varying concentration of the appropriate inducer. These culture plates were then grown in a Pro200 (Tecan) microplate reader at $30^{\circ} \mathrm{C}, 37 \mathrm{rpm}$, for 72 hours. $\mathrm{OD}_{600}$ and RFP fluorescence intensity, using an excitation wavelength of $585 \mathrm{~nm}$ and an emission wavelength of $620 \mathrm{~nm}$, were measured after 48 hours.

\section{Hydrocarbon production assay}

Single colonies were picked and inoculated into $10 \mathrm{~mL}$ fresh LB glass culture tubes and grown at $30^{\circ} \mathrm{C}$ for 15 hours. The appropriate inducer was then added to each culture tube (final concentration: $1 \mathrm{mM}$ IPTG, 0.1\% Larabinose, or $1 \mathrm{mM}$-toluic acid). $1 \mathrm{~mL}$ decane (Sigma, 99\% purity) was then immediately added to each $10 \mathrm{~mL}$ culture tube to extract hydrocarbons and other metabolites. 72 hours after induction, $100 \mu \mathrm{L}$ of each decane overlay was removed for direct gas chromatography-mass spectrometry (GC/MS) analysis. Electron ionization (EI) GC/MS analyses were performed with a model 7890A GC quadrupole mass spectrometer (Agilent) with a DB-5 fused silica capillary column ( $\mathrm{J} \& \mathrm{~W}$ Scientific, 30-m length, $0.25-\mathrm{mm}$ inner diameter, $0.25-\mu \mathrm{m}$ film thickness) coupled to a HP 5975C mass selective detector. $1 \mu \mathrm{L}$ injections were performed by an Agilent model 7683B autosampler. The GC oven was typically programmed to ramp from $40^{\circ} \mathrm{C}$ (held for 3 minutes) to $300^{\circ} \mathrm{C}$ at $15^{\circ} \mathrm{C} / \mathrm{min}$ and then held for 5 minutes. The injection port temperature was $250^{\circ} \mathrm{C}$, and the transfer line temperature was $280^{\circ} \mathrm{C}$. The carrier gas, ultra-high purity helium, flowed at a constant rate of $1 \mathrm{~mL} / \mathrm{min}$. Injections were splitless, with the split turned on after 0.5 minutes. For full-scan data acquisition, the MS typically scanned from 50 to 600 atomic mass units at a rate of 2.7 scans per second. One of the major products, pentadecane (15:0), was quantified with 
$m / z 57$ area, while the other major product, heptadecene (17:1), was quantified with $m / z 83$ area of authentic standards (Sigma, 99\% purity).

\section{Additional file}

\section{Additional file 1: Supplementary Materials and Methods.}

\section{Abbreviations}

R. eutropha: Ralstonia eutropha; PHB: Polyhydroxybutyrate; RBS: Ribosomal binding site; LB: Lysogeny broth.

\section{Competing interests}

The authors declare that they have no competing interests.

\section{Authors' contributions}

CB designed and carried out this work, and drafted the manuscript. PS participated in experimental design, and molecular genetic studies and other experimental aspects of this work. JM participated in experimental design and strain construction. YY participated in genetic studies. SRC, HRB, and SWS supervised the research and edited the manuscript. NJH supervised the research, and wrote and edited the manuscript. All authors read and approved the final version of the manuscript.

\section{Acknowledgements}

This work was funded by the Department of Energy, Advanced Research Projects Agency-Energy (ARPA-E) Electrofuels Program, under contract DE0000206-1577 to Lawrence Berkeley National Laboratory. This work was performed at the Joint BioEnergy Institute, which is funded by the Department of Energy, Office of Science, Office of Biological and Environmental Research under contract DE-AC02-5CH11231 to Lawrence Berkeley National Laboratory. We thank Vivek Mutalik for providing access to the Guava easyCyte Flow cytometry system. We thank Jonathan Vroom for assistance in constructing trfA mutations and the T7 stem-loop. We thank Yung Hsu Tang for assistance in constructing and testing pKTTrfp, pCMTrfp, pCM271Trfp, and pCM271TcalRBSrfp.

\section{Author details}

${ }^{1}$ Physical Biosciences Division, Lawrence Berkeley National Laboratory, Berkeley, CA 94720, USA. ${ }^{2}$ Present address: Tianjin Institute of Biotechnology, Chinese Academy of Sciences, Tianjin, China. ${ }^{3}$ Department of Chemical \& Biomolecular Engineering, University of California, Berkeley, CA 94720, USA. ${ }^{4}$ National Taiwan Normal University, Taipei, Taiwan. ${ }^{5}$ Earth Sciences Division, Lawrence Berkeley National Laboratory, Berkeley, CA 94720, USA.

Received: 13 September 2013 Accepted: 11 November 2013 Published: 13 November 2013

\section{References}

1. Müller J, MacEachran D, Burd H, Sathitsuksanoh N, Bi C, Yeh YC, Lee TS, Hillson NJ, Chhabra SR, Singer SW, Beller HR: Engineering of Ralstonia eutropha $\mathrm{H} 16$ for autotrophic and heterotrophic production of methyl ketones. Appl Environ Microbiol 2013, 79:4433-4439.

2. Li H, Opgenorth PH, Wernick DG, Rogers S, Wu TY, Higashide W, Malati P, Huo YX, Cho KM, Liao JC: Integrated electromicrobial conversion of $\mathrm{CO}_{2}$ to higher alcohols. Science 2012, 335:1596.

3. Reinecke F, Steinbuchel A: Ralstonia eutropha strain $\mathrm{H} 16$ as model organism for PHA metabolism and for biotechnological production of technically interesting biopolymers. J Mol Microbiol Biotechnol 2009, 16:91-108.

4. Ishizaki A, Tanaka K, Taga N: Microbial production of poly-D-3-hydroxybutyrate from $\mathrm{CO}_{2}$. Appl Microbiol Biotechnol 2001, 57:6-12

5. Lenz O, Friedrich $B$ : A novel multicomponent regulatory system mediates $\mathrm{H}_{2}$ sensing in Alcaligenes eutrophus. Proc Natl Acad Sci USA 1998, 95:12474-12479.

6. Fodor BD, Kovacs AT, Csaki R, Hunyadi-Gulyas E, Klement E, Maroti G, Meszaros LS, Medzihradszky KF, Rakhely G, Kovacs KL: Modular broad-host-range expression vectors for single-protein and protein complex purification. Appl Environ Microbiol 2004, 70:712-721.
7. Delamarre SC, Batt CA: Comparative study of promoters for the production of polyhydroxyalkanoates in recombinant strains of Wautersia eutropha. Appl Microbiol Biotechnol 2006, 71:668-679.

8. Marx CJ, Lidstrom ME: Development of improved versatile broad-host-range vectors for use in methylotrophs and other Gram-negative bacteria. Microbiology-Sgm 2001, 147:2065-2075.

9. Kovach ME, Phillips RW, Elzer PH, Roop RM 2nd, Peterson KM: pBBR1MCS: a broad-host-range cloning vector. Biotechniques 1994, 16:800-802.

10. Sode K, Tatara M, Ogawa S, Matsunaga T: Maintenance of broad host range vector Pkt230 in marine unicellular cyanobacteria. Fems Microbiology Letters 1992, 99:73-78.

11. Brigham CJ, Budde CF, Holder JW, Zeng Q, Mahan AE, Rha C, Sinskey AJ: Elucidation of beta-oxidation pathways in Ralstonia eutropha $\mathrm{H} 16$ by examination of global gene expression. J Bacterio/ 2010, 192:5454-5464.

12. Simon $R$, Priefer $U$, Puhler $A$ : A broad host range mobilization system for invivo genetic-engineering - transposon mutagenesis in gram-negative bacteria. Bio/Technology 1983, 1:784-791.

13. Yeh YC, Muller J, Bi C, Hillson NJ, Beller HR, Chhabra SR, Singer SW: Functionalizing bacterial cell surfaces with a phage protein. Chem Commun (Camb) 2013, 49:910-912.

14. Lee TS, Krupa RA, Zhang F, Hajimorad M, Holtz WJ, Prasad N, Lee SK, Keasling JD: BglBrick vectors and datasheets: a synthetic biology platform for gene expression. J Biol Eng 2011, 5:12

15. Durland RH, Toukdarian A, Fang F, Helinski DR: Mutations in the trfA replication gene of the broad-host-range plasmid RK2 result in elevated plasmid copy numbers. J Bacteriol 1990, 172:3859-3867.

16. Haugan K, Karunakaran P, Tondervik A, Valla S: The host range of RK2 minimal replicon copy-up mutants is limited by species-specific differences in the maximum tolerable copy number. Plasmid 1995, 33:27-39.

17. Mertens N, Remaut E, Fiers W: Increased stability of phage T7g10 mRNA is mediated by either a 5'- or a 3'-terminal stem-loop structure. Biol Chem 1996, 377:811-817.

18. Fukui T, Ohsawa K, Mifune J, Orita I, Nakamura S: Evaluation of promoters for gene expression in polyhydroxyalkanoate-producing Cupriavidus necator H16. Appl Microbiol Biotechnol 2011, 89:1527-1536.

19. Hansen LH, Knudsen S, Sorensen SJ: The effect of the lacY gene on the induction of IPTG inducible promoters, studied in Escherichia coli and Pseudomonas fluorescens. Curr Microbiol 1998, 36:341-347.

20. Salis HM, Mirsky EA, Voigt CA: Automated design of synthetic ribosome binding sites to control protein expression. Nat Biotechno/ 2009, 27:946-950.

21. Schirmer A, Rude MA, Li X, Popova E, del Cardayre SB: Microbial biosynthesis of alkanes. Science 2010, 329:559-562.

22. Mutalik VK, Guimaraes JC, Cambray G, Lam C, Christoffersen MJ, Mai QA, Tran AB, Paull M, Keasling JD, Arkin AP, Endy D: Precise and reliable gene expression via standard transcription and translation initiation elements. Nat Methods 2013, 10:354-360.

23. Ma SM, Garcia DE, Redding-Johanson AM, Friedland GD, Chan R, Batth TS, Haliburton JR, Chivian D, Keasling JD, Petzold CJ, et al: Optimization of a heterologous mevalonate pathway through the use of variant HMG-CoA reductases. Metab Eng 2011, 13:588-597.

24. Redding-Johanson AM, Batth TS, Chan R, Krupa R, Szmidt HL, Adams PD, Keasling JD, Lee TS, Mukhopadhyay A, Petzold CJ: Targeted proteomics for metabolic pathway optimization: application to terpene production. Metab Eng 2011, 13:194-203.

25. Zhao DL, Yu ZC, Li PY, Wu ZY, Chen XL, Shi M, Yu Y, Chen B, Zhou BC, Zhang YZ: Characterization of a cryptic plasmid pSM429 and its application for heterologous expression in psychrophilic Pseudoalteromonas. Microb Cell Fact 2011, 10:30.

26. Dammeyer T, Steinwand M, Kruger SC, Dubel S, Hust M, Timmis KN. Efficient production of soluble recombinant single chain Fv fragments by a Pseudomonas putida strain KT2440 cell factory. Microb Cell Fact 2011, 10:11.

27. Dammeyer T, Timmis KN, Tinnefeld P: Broad host range vectors for expression of proteins with (Twin-) Strep-tag, His-tag and engineered, export optimized yellow fluorescent protein. Microb Cell Fact 2013, 12:49.

28. Quan J, Tian J: Circular polymerase extension cloning for high-throughput cloning of complex and combinatorial DNA libraries. Nat Protoc 2011, 6:242-251.

29. Gibson DG, Young L, Chuang RY, Venter JC, Hutchison CA 3rd, Smith HO: Enzymatic assembly of DNA molecules up to several hundred kilobases. Nat Methods 2009, 6:343-345. 
30. Hillson NJ, Rosengarten RD, Keasling JD: j5 DNA assembly design automation software. ACS Synth Biol 2012, 1:14-21.

31. Chen J, Densmore D, Ham TS, Keasling JD, Hillson NJ: DeviceEditor visual biological CAD canvas. J Biol Eng 2012, 6:1.

32. Linshiz G, Stawski N, Poust S, Bi C, Keasling JD, Hillson NJ: PaR-PaR laboratory automation platform. ACS Synth Biol 2013, 2:216-222

33. Ham TS, Dmytriv Z, Plahar H, Chen J, Hillson NJ, Keasling JD: Design, implementation and practice of JBEI-ICE: an open source biological part registry platform and tools. Nucleic Acids Res 2012, 40:e141.

34. Campbell RE, Tour O, Palmer AE, Steinbach PA, Baird GS, Zacharias DA,

Tsien RY: A monomeric red fluorescent protein. Proc Natl Acad Sci USA 2002, 99:7877-7882

doi:10.1186/1475-2859-12-107

Cite this article as: Bi et al:: Development of a broad-host synthetic

biology toolbox for ralstonia eutropha and its application to engineering hydrocarbon biofuel production. Microbial Cell Factories 2013 12:107.

\section{Submit your next manuscript to BioMed Central and take full advantage of:}

- Convenient online submission

- Thorough peer review

- No space constraints or color figure charges

- Immediate publication on acceptance

- Inclusion in PubMed, CAS, Scopus and Google Scholar

- Research which is freely available for redistribution 